\title{
THE INFLUENZA AH1N1: A MATHEMATICAL MODEL WITH APPLICATION OF OPTIMAL CONTROL
}

\author{
Elmer De la Pava $^{1} \S$, Beatriz Salguero ${ }^{2}$, Lilian Sofía Sepúlveda ${ }^{3}$ \\ $1,2,3$ Department of Mathematics \\ Universidad Autónoma de Occidente \\ Cali, COLOMBIA
}

\begin{abstract}
Human influenza is transmitted directly from an ill person to a healthy one, by air, during the symptomatic period of the disease. The virulence and antigenicity of the virus, host immunity and environment, interact with each other affecting the transmission of the virus person-person. Since the alert in 2009 of the influenza due to the H1N1 virus, the number of new cases increased despite the control measures implement, such as wearing masks, and other recommendations made by the World Health Organization. In Colombia it was found that the first case of AH1N1 coincided with a person from Mexico. Therefore, and considering that all travelers from this country are a suspected case of the disease, it makes the transit from one country to another a possible route of transmission. Hence, the approach to the question: What has been the impact in Colombia of the outbreak caused by the migration of people from Mexico infected with the H1N1 influenza virus?

The porpuse of this paper is, in a certain way, to respond this question using a mathematical model that studies the transmission of this disease in both immigrant and local populations. Population $N$ is divided in $N_{E}$ immigrant populations and $N_{L}$ local population, where $N=N_{E}+N_{L}$, according to the natural history of H1N1. Each subpopulation is divided into three classes, susceptible $S$, infectious $I$ and recovered $R$, resulting in the six compartments $S_{E}, I_{E}, R_{E}$ and $S_{L}, I_{L}, R_{L}$. The equilibriums and their qualitative analysis were calculated. Besides, the basic reproductive number representing the classical measure of transmission of infectious diseases is estimated and, from a biological point of view, it is defined as the number of secondary cases produced by a typical infected individual when introduced in a fully susceptible host population, during its effective period of infectivity. If
\end{abstract}

Received: January 13, 2016

Published: February 27, 2016

$\S_{\text {Correspondence author }}$ (c) 2016 Academic Publications, Ltd.

url: www.acadpubl.eu 
$\mathcal{R}_{0}<1$ the disease goes out and if $\mathcal{R}_{0}>1$ an outbreak occurs.

AMS Subject Classification: 34H05, 49J15, 49K15, 93C15

Key Words: model, control, influenza, infectious

\section{Introduction}

There are three types of influenza virus: A, B and C. Types A and B are more common in humans [1]. The epidemiology of type A differs significantly from that of type B or C. Type A induces higher levels of disease in humans and circulates in animal populations such as birds, pigs and horses. Types B and $\mathrm{C}$ viruses propagate only among humans. The influenza $\mathrm{A}$ and $\mathrm{B}$ have the capacity to improve their ability to invade the host population through minor mutations (antigenic drift). Major mutations (antigenic shift) are possible and are assumed to be responsible for major type A pandemics [[1], [2]]. Types $\mathrm{B}$ and $\mathrm{C}$ viruses propagate only among humans. Type $\mathrm{A}$ are classified into three subtypes according to the characterization of their highly variable surface antigens hemagglutinin (HA) and neuraminidase (NA). These subtypes are known as H1N1, H2N2 and H3N2 where $\mathrm{H}$ and $\mathrm{N}$ describe specific hemagglutinin and neuraminidase molecules, respectively. Major mutations in HA and/or NA molecules (antigenic shift) involve the replacement of one or both of the $\mathrm{H}$ and $\mathrm{N}$ antigens. For instance, the displacement at the population level of the H1N1 subtype by H2N2 in 1968, provides one example. Several theories have been given to explain the origin of these mutations; however, the most common argues that re-assortment of the virus genetic material in human and animals is highly possible given the large reservoir of flu A viruses in animal populations. Aside from characterizing type A viruses into subtypes, minor mutations (antigenic drift) within each of these subtypes are classified as variants or subtype specific strains. These new strains facilitate the invasion of the host population on a yearly basis, regardless of the levels immunity or crossimmunity acquired from prior infections [[3], [4]]. Influenza strains are classified according to their type (A or B), town of isolation, number of isolates, year of isolation and subtypes (HA and NA), and strain [4].

Mathematical models have been an important tool in the analyzis of infectious diseases [5]. In 1760 Daniel Bernoulli formulated and solved a model for smallpox [5]. In 1906, Hamer [6] posed and analyzed a discrete model that attempts to understand the recurrence of epidemics of measles. In 1911, Ross [7] was interested in the incidence and control of malaria and developed 
a host-parasite model based on differential equations. in 1926, Kermack and McKendrick [8] published an article on epidemic models. Since then, research in epidemiology mathematics has grown almost exponentially, and a variety of models have been mathematically analyzed and applied to infectious diseases [[9], [10], [11], [12]]. Today, there are thousands contributions, with magazines almost exclusively devoted to such topics.

Different types of models have been proposed to describe the outbreaks of influenza. Many public health policies to address with pandemic influenza are built based on networks of contact in a population and the propagation of the disease taking place through this network. These tests consist of multiple stochastic simulations that require a lot of time on the computer. In this research we intend to work using simple models with ordinary differential equations, and collect current data to estimate the important parameters of these models [11].

We formulate an optimal control model for the transmission of the influenza $\mathrm{H} 1 \mathrm{~N} 1$ in immigrants and local population, in order to derive optimal prevention and treatment strategies with minimal implementation cost. We consider (time dependent) optimal control strategies associated with two time-dependent controls. The first control, $u_{1}(t)$ is applied to the transmission rate, and represents the insulation made of the cases presented. The second control, $u_{2}(t)$, represents the effort put into the treatment of individuals infected with H1N1.

\section{Model}

In order to study the transmission of the disease in immigrants and local population, the total population $N$ has been divided into $N_{E}$ immigrant population and $N_{L}$ locals, where $N=N_{E}+N_{L}$ according to the natural history of the disease. Each subpopulation is divided into three classes: susceptible $S$, infectious $I$ and recovered $R$, this resulting into the six compartments $S_{E}, I_{E}, R_{E}$ and $S_{L}, I_{L}, R_{L}$, whose flowchart is shown in Figure 1, and where the influence of local active immigrant population immigrants is negligible. The model is specified by six differential equations: 


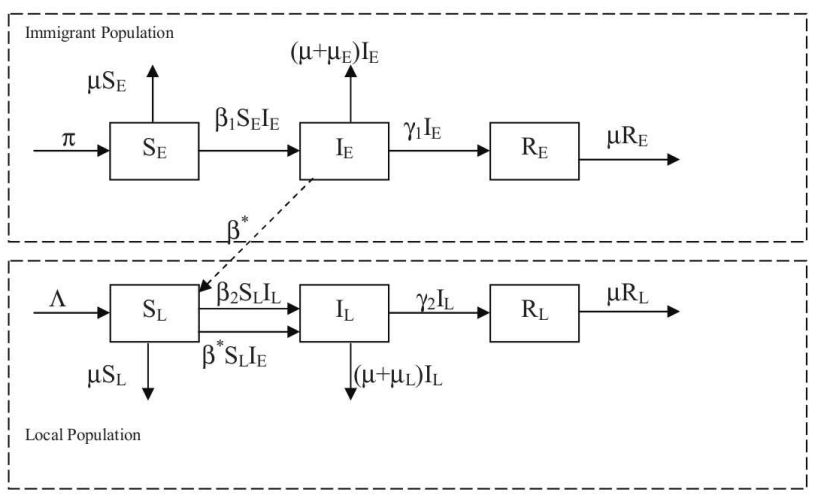

Figure 1: diagram AH1N1

$$
\begin{aligned}
S_{E}^{\prime}(t) & =\pi-\beta_{1} S_{E}(t) I_{E}(t)-\mu S_{E}(t), \\
I_{E}^{\prime}(t) & =\beta_{1} S_{E}(t) I_{E}(t)-\left(\gamma_{1}+\mu+\mu_{I_{E}}\right) I_{E}(t) \\
R_{E}^{\prime}(t) & =\gamma_{1} I_{E}(t)-\mu R_{E}(t), \\
N_{E} & =S_{E}(t)+I_{E}(t)+R_{E}(t),
\end{aligned}
$$

and

$$
\begin{aligned}
S_{L}^{\prime}(t) & =\Lambda-\beta_{2} S_{L}(t) I_{L}(t)-\beta^{*} S_{L}(t) I_{E}(t)-\mu S_{L}(t), \\
I_{L}^{\prime}(t) & =\beta_{2} S_{L}(t) I_{L}(t)+\beta^{*} S_{L}(t) I_{E}(t)-\left(\gamma_{2}+\mu+\mu_{I_{L}}\right) I_{L}(t) \\
R_{L}^{\prime}(t) & =\gamma_{2} I_{L}(t)-\mu R_{L}(t), \\
N_{L} & =S_{L}(t)+I_{L}(t)+R_{L}(t),
\end{aligned}
$$

where $\pi$ and $\Lambda$ are constant recruitment within populations $N_{E}, N_{L}$ respectively, $\beta_{1}$ and $\beta_{2}$ are the transmission coefficients for the populations of immigrants and local population. $\beta^{*}$ is the coefficient for the transmission of the disease, from immigrants to the susceptible local population . In this model, the term $\beta^{*} S_{L}(t) I_{E}(t)$ models the impact of the immigrant population infected with H1N1 within the local population. 


\section{Global Stability of Disease-Free Equilibrium}

Domains

$$
\Omega_{E}=\left\{\left(S_{E}, I_{E}, R_{E}\right) \in \mathbb{R}^{3}: S_{E}, I_{E}, R_{E} \geq 0, S_{E}+I_{E}+R_{E} \leq \frac{\Lambda}{\mu}\right\}
$$

and

$$
\Omega_{L}=\left\{\left(S_{L}, I_{L}, R_{L}\right) \in \mathbb{R}^{3}: S_{L}, I_{L}, R_{L} \geq 0, S_{L}+I_{L}+R_{L} \leq \frac{\Lambda}{\mu}\right\}
$$

are positive and invariant because they are constrained by the invariant planes $S_{E}=0, I_{E}=0, R_{E}=0, S_{L}=0, I_{L}=0, R_{L}=0$, and the planes $S_{E}+I_{E}+R_{E}=$ $\frac{\Lambda}{\mu}, S_{L}+I_{L}+R_{L}=\frac{\Lambda}{\mu}$. In other planes that limit the region entering the orbits only, as discussed below.

In the plane $S_{E}=0$ the first equation for system (1) is equal to $\frac{d S_{E}}{d t}=\pi$, orbits cross the plane, entering the region $\omega_{E}$. In the plane $I_{E}=0$ the second equation for system (1) is equal to $\frac{d I_{E}}{d t}=0$, that is, the orbits of the plane do not leave him, neither does the plane $R_{E}=0$, then $\frac{d R_{E}}{d t}=0$.

\subsection{Basic Reproductive Number}

(1) and (2) we obtain the following trivial equilibria:

$$
P_{E 0}=\left(\frac{\pi}{\mu}, 0,0\right), \quad P_{L 0}=\left(\frac{\pi}{\mu}, 0,0\right)
$$

The Jacobian matrix of the system(1) is

$$
J=\left(\begin{array}{ccc}
-\beta_{1} I_{E}-\mu & -\beta_{1} S_{E} & 0 \\
\beta_{1} I_{E} & \beta_{1} S_{E}-\left(\gamma_{1}+\mu+\mu_{I_{E}}\right) & 0 \\
0 & \gamma_{1} & -\mu
\end{array}\right) .
$$

Then

$$
J\left(P_{E 0}\right)=\left(\begin{array}{ccc}
-\mu & -\beta_{1} \frac{\pi}{\mu} & 0 \\
0 & \beta_{1} S_{E}-\left(\gamma_{1}+\mu+\mu_{I_{E}}\right) & 0 \\
0 & \gamma_{1} & -\mu
\end{array}\right) .
$$

Then, from the operator of the next generation in the matrix $J\left(P_{E 0}\right)$, we obtain the following matrices

$$
M=\left(\begin{array}{ccc}
0 & -\beta_{1} \frac{\pi}{\mu} & 0 \\
0 & \beta_{1} \frac{\pi}{\mu} & 0 \\
0 & \gamma_{1} & -\mu
\end{array}\right) ; \quad D=\left(\begin{array}{ccc}
\mu & 0 & 0 \\
0 & \gamma_{1}+\mu+\mu_{I_{E}} & 0 \\
0 & 0 & \mu
\end{array}\right)
$$


and

$$
A=M D^{-1}=\left(\begin{array}{ccc}
0 & -\frac{\beta_{1} \pi}{\mu\left(\gamma_{1}+\mu+\mu_{I_{E}}\right)} & 0 \\
0 & \frac{\beta_{1} \pi}{\mu\left(\gamma_{1}+\mu+\mu_{I_{E}}\right)} & 0 \\
0 & 0 & 0
\end{array}\right)
$$

and the characteristic polynomial of matrix $A$ is

$$
P(\lambda)=\lambda^{2}\left(\lambda-\frac{\beta_{1} \pi}{\mu\left(\gamma_{1}+\mu+\mu_{I_{E}}\right)}\right)=0
$$

and the largest eigenvalue is the basic reproductive number for the immigrant population given by

$$
\mathcal{R}_{E 0}=\frac{\beta_{1} \pi}{\mu\left(\gamma_{1}+\mu+\mu_{I_{E}}\right)} .
$$

With a similar procedure we obtain the basic reproductive number for the local population given by

$$
\mathcal{R}_{L 0}=\frac{\beta_{2} \Lambda}{\mu\left(\gamma_{2}+\mu+\mu_{I_{L}}\right)} .
$$

Then,

$$
\mathcal{R}_{0}=\max \left\{\mathcal{R}_{E 0}, \mathcal{R}_{L 0}\right\}
$$

the number of secondary cases produced when an infectious individual is introduced into a susceptible population during its infectious period. The disease would disappear if $\mathcal{R}_{0}<1$ or would become epidemic if $\mathcal{R}_{0}>1$. Directly from the system (1) it can be shown that when $\mathcal{R}_{0}<1$ there is only one disease-free equilibrium given by

$$
P_{0}=\left(P_{E 0}, P_{L 0}\right)
$$

as shown in the following theorem.

Theorem 1. If $\mathcal{R}_{0}<1$, that is, when $\mathcal{R}_{E 0} \leq 1$ and $\mathcal{R}_{L 0} \leq 1$ then $P_{0}$ is the unique equilibrium of the system (1), (2) and is globally asymptotically stable in $\Omega$. Si $\mathcal{R}_{0}>1$, then $P_{0}$ is unstable. Where $\Omega=\Omega_{E}+\Omega_{L}$.

Proof. For the system of differential equations (1), we have

$$
V_{E}=I_{E}
$$

Then

$$
\frac{d V_{E}}{d t}=\frac{\beta_{1} \pi}{\mu}\left(\frac{\mu}{\pi} S_{E}-\frac{1}{\mathcal{R}_{0}}\right) I_{E} \leq 0,
$$

if $\mathcal{R}_{E 0}<1$. Also, $\frac{d V_{E}}{d t}=0$ iff $I_{E}=0$. The maximum set invariant and compact in $\Omega$ is determined solely by $\left\{P_{E 0}\right\}$. Based on Lasalle invariance principle, $P_{E 0}$ 
is globally asymptotically stable in $\Omega$. Since it is assumed that $I_{E}=0$ as $t \longrightarrow 0$, the system $(2)$ is rewritten as

$$
\begin{aligned}
S_{L}^{\prime}(t) & =\Lambda-\beta_{2} S_{L}(t) I_{L}(t)-\mu S_{L}(t) \\
I_{L}^{\prime}(t) & =\beta_{2} S_{L}(t) I_{L}(t)-\left(\gamma_{2}+\mu+\mu_{I_{L}}\right) I_{L}(t) \\
R_{L}^{\prime}(t) & =\gamma_{2} I_{L}(t)-\mu R_{L}(t) \\
N_{L} & =S_{L}(t)+I_{L}(t)+R_{L}(t)
\end{aligned}
$$

then similarly for system (1) we have the function

$$
V_{L}=I_{L}
$$

Therefore,

$$
\frac{d V_{L}}{d t}=\frac{\beta_{2} \Lambda}{\mu}\left(\frac{\mu}{\Lambda} S_{L}-\frac{1}{\mathcal{R}_{L 0}}\right) I_{L} \leq 0,
$$

if $\mathcal{R}_{L 0}<1$. Similar to $P_{E 0}, P_{L 0}$ is globally asymptotically stable. In conclusion, $P_{0}=\left(P_{E 0}, P_{L 0}\right)$ is globally asymptotically stable in $\Omega=\Omega_{E}+\Omega_{L}$ si $\mathcal{R}_{0}<1$.

\subsection{Endemic Equilibrium}

Theorem 2. If $\mathcal{R}_{0}>1$, then

i) If $\mathcal{R}_{E 0}>1$, independently of $\mathcal{R}_{L 0}$, system (1), (2) has a unique endemic equilibrium $P^{*}=\left(P_{E}^{*}, P_{L}^{*}\right)$ in $\Omega$.

ii) If $\mathcal{R}_{E 0} \leq 1$ y $\mathcal{R}_{L 0}>1$ system (1) y (2) has a unique equilibrium $P^{\prime}=$ $\left(P_{E 0}, P_{L}^{*}\right)$ in $\Omega$.

Proof. Equating to zero each equation of the system (1) and (2) we get

$$
\begin{aligned}
\pi-\beta_{1} S_{E}(t) I_{E}(t)-\mu S_{E}(t) & =0, \\
\beta_{1} S_{E}(t) I_{E}(t)-\left(\gamma_{1}+\mu+\mu_{I_{E}}\right) I_{E}(t) & =0, \\
\gamma_{1} I_{E}(t)-\mu R_{E}(t) & =0, \\
\Lambda-\beta_{2} S_{L}(t) I_{L}(t)-\beta^{*} S_{L}(t) I_{E}(t)-\mu S_{L}(t) & =0, \\
\beta_{2} S_{L}(t) I_{L}(t)+\beta^{*} S_{L}(t) I_{E}(t)-\left(\gamma_{2}+\mu+\mu_{I_{L}}\right) I_{L}(t) & =0 \\
\gamma_{2} I_{L}(t)-\mu R_{L}(t) & =0 .
\end{aligned}
$$


Calculating directly from (3) we have for the system (1) that the endemic equilibrium is given by the following expressions

$$
S_{E}^{*}=\frac{\pi}{\mu} \mathcal{R}_{E 0} ; \quad I_{E}^{*}=\left(\mathcal{R}_{E 0}-1\right) \frac{\mu}{\beta_{1}} ; \quad I_{E}^{*}=\left(\mathcal{R}_{E 0}-1\right) \frac{\gamma_{1}}{\beta_{1}}
$$

and for the system (2) the endemic equilibrium is given by

$$
S_{L}^{*}=\frac{\Lambda}{\mu \mathcal{R}_{L 0}} ; \quad I_{L}^{*}=\left(\mathcal{R}_{L 0}-1\right) \frac{\mu}{\beta_{2}} ; \quad I_{L}^{*}=\left(\mathcal{R}_{L 0}-1\right) \frac{\gamma_{2}}{\beta_{2}} .
$$

This implies that there is an endemic equilibrium of the form

$$
P^{*}=\left(P_{E}^{*}, P_{L}^{*}\right)
$$

where $P_{M}^{*}=\left(S_{E}^{*}, I_{E}^{*}, R_{E}^{*}\right)$ y $P_{L}^{*}=\left(S_{L}^{*}, I_{L}^{*}, R_{L}^{*}\right)$

\section{Control Model}

Control theory can help determine how to produce maximum performance or minimal cost of the measures taken to control an epidemic. In model (1)we consider two time-dependent controls. The first control, $u_{1}(t)$, is applied to the transmission rate $\beta_{2}$, and represents the insulation made of the cases presented. The second control, $u_{2}(t)$, represents the effort put into the treatment of individuals infected with H1N1 [13]. Then, adding these controls in the system (1) yields the following system of differential equations with control:

$$
\begin{aligned}
S_{L}^{\prime}(t)= & \Lambda-\beta_{2}\left(1-u_{1}(t)\right) S_{L}(t) I_{L}(t)-\beta^{*} S_{L}(t) I_{E}(t)-\mu S_{L}(t), \\
I_{L}^{\prime}(t)= & \beta_{2}\left(1-u_{1}(t)\right) S_{L}(t) I_{L}(t)+\beta^{*} S_{L}(t) I_{E}(t)-\left(\gamma_{2}+\gamma_{3} u_{2}(t)\right. \\
& \left.+\mu+\mu_{I_{L}}\right) I_{L}(t), \\
R_{L}^{\prime}(t)= & \left(\gamma_{2}+\gamma_{3} u_{2}(t)\right) I_{L}(t)-\mu R_{L}(t),
\end{aligned}
$$

where $N_{L}=S_{L}(t)+I_{L}(t)+R_{L}(t)$. The utility in implementing these control measures is always very beneficial; however, each measure brings about cost control, social isolation, creates economic losses, while the treatment requires adequate supply infrastructure [13]. Since the coefficient $\left(1-u_{1}(t)\right)$ represents the effect of preventing new infections with the isolation of infected persons, when $u_{1}(t)$ is close to 1 , there is a decline in new cases, but the costs of implementing this control are high. We assume that these costs are nonlinear and are square. 
The functional objective to be minimized is

$$
J\left(u_{1}, u_{2}\right)=\int_{0}^{t_{f}}\left[I_{L}(t)+\frac{A_{1}}{2} u_{1}^{2}(t)+\frac{A_{2}}{2} u_{2}^{2}(t)\right] d t
$$

where we want to minimize the infectious group and keep the cost of treatment low. The coefficients $A_{1}$ and $A_{2}$ are balancing the cost factors of each control. The goal is to find a pair of controls $\left(u_{1}^{*}, u_{2}^{*}\right)$, such that

$$
J\left(u_{1}^{*}, u_{2}^{*}\right)=\min _{\Omega} J\left(u_{1}, u_{2}\right)
$$

where

$$
\Omega=\left\{\left(u_{1}, u_{2}\right) \in L^{1}\left(0, t_{f}\right) \mid a_{i} \leq u_{i} \leq b_{i}, i=1,2\right\},
$$

$a_{i}, b_{i}, i=1,2$, are positive constants.

\subsection{Analysis of Optimal Control}

The conditions necessary so that a pair of controls $\left(u_{1}^{*}, u_{2}^{*}\right)$ is optimal comes from the Pontryagin Maximum Principle [3]. This principle turns the system (4)-(6) into a minimization problem of a Hamiltonian, $H$, with respect to $u_{1} \mathrm{y}$ $u_{2}$ :

$$
\begin{aligned}
H & =I_{L}+\frac{A_{1}}{2} u_{1}^{2}(t)+\frac{A_{2}}{2} u_{2}^{2}(t) \\
& +\lambda_{1}\left(\Lambda-\beta_{2}\left(1-u_{1}(t)\right) S_{L}(t) I_{L}(t)-\beta^{*} S_{L}(t) I_{E}(t)-\mu S_{L}(t)\right) \\
& +\lambda_{2}\left[\beta_{2}\left(1-u_{1}(t)\right) S_{L}(t) I_{L}(t)+\beta^{*} S_{L}(t) I_{E}(t)\right. \\
& \left.-\left(\gamma_{2}+\gamma_{3} u_{2}(t)+\mu+\mu_{I_{L}}\right) I_{L}(t)\right]+\lambda_{3}\left(\left(\gamma_{2}+\gamma_{3} u_{2}(t)\right) I_{L}(t)-\mu R_{L}(t)\right) .
\end{aligned}
$$

Based on the Pontryagin Maximum Principle [3] and the existence result for a pair of controls $\left(u_{1}^{*}, u_{2}^{*}\right)[3]$, we formulated the following theorem

Theorem 3. There is an optimal control pair $\left(u_{1}^{*}, u_{2}^{*}\right)$ and their solutions $\left(S_{L}^{*}, I_{L}^{*}, R_{L}^{*}\right)$ minimizing $J\left(u_{1}, u_{2}\right)$ in $\Omega$. In addition, there are functions $\lambda_{1}(t)$, $\lambda_{2}(t), \lambda_{3}(t)$, such that

$$
\begin{aligned}
\frac{d \lambda_{1}}{d t}= & \lambda_{1}\left[\beta_{2}\left(1-u_{1}(t)\right) I_{L}+\beta^{*} I_{E}(t)-\mu\right]-\lambda_{2}\left[\beta_{2}\left(1-u_{1}(t)\right) I_{L}(t)+\beta^{*} I_{E}(t)\right] \\
\frac{d \lambda_{2}}{d t}= & -1+\lambda_{1} \beta_{2}\left(1-u_{1}(t)\right) S_{L}(t) \\
& -\lambda_{2}\left[\beta_{2}\left(1-u_{1}(t)\right) S_{L}-\left(\gamma_{2}+\gamma_{3} u_{2}(t)+\mu+\mu_{I_{L}}\right)\right]-\lambda_{3}\left[\gamma_{2}+\gamma_{3} u_{2}(t)\right], \\
\frac{d \lambda_{3}}{d t}= & \mu
\end{aligned}
$$


with transversality conditions

$$
\lambda_{i}\left(t_{f}\right)=0 \quad \text { para } i=1,2,3 .
$$

Also, the following inequalities are valid

$$
\begin{gathered}
u_{1}^{*}=\min \left(\max \left(L A_{1}, \frac{1}{A_{1}}\left[\left(\lambda_{2}-\lambda_{1}\right) \beta_{2} S_{L} I_{L}\right]\right), U A_{1}\right) \\
u_{2}^{*}=\min \left(\max \left(L A_{2}, \frac{1}{A_{2}}\left[\left(\lambda_{2}-\lambda_{3}\right) \gamma_{3} I_{L}\right]\right), U A_{2}\right)
\end{gathered}
$$

where $L A_{i}$ is the lower bound for $u_{i}$, and $U A_{i}$, is the upper bound for $u_{i}$, $i=1,2$.

Proof. In the corollary, 4.1 of [14] Fleming et al. shows that there is a pair of optimal control due to the convexity of the integrand of $J$ in (5) with respect to $\left(u_{1}, u_{2}\right)$, a priori bound of solutions and system (4) is Lipshitz with respect to state variables. The following relationships are obtained directly from the Pontryagin Maximum Principle [3]:

$$
\frac{d \lambda_{1}}{d t}=-\frac{\partial H}{\partial S_{L}}, \quad \frac{d \lambda_{2}}{d t}=-\frac{\partial H}{\partial I_{L}}, \quad \frac{d \lambda_{3}}{d t}=-\frac{\partial H}{\partial R_{L}},
$$

with $\lambda_{i}\left(t_{f}\right)=0$ for $i=1,2,3$ and evaluated the optimal control pair and their corresponding states. Here, the systems (8) and (9) have been deduced. The Hamiltonian $H$ must be minimized with respect to the pair of controls $\left(u_{1}, u_{2}\right)$ on the set $\Omega$. From these calculations we obtain

$$
\begin{gathered}
\frac{\partial H}{\partial u_{1}}=A_{1} u_{1}+\left(\lambda_{1}-\lambda_{2}\right) \beta_{2} S_{L} I_{L}=0 \\
\frac{\partial H}{\partial u_{2}}=A_{2} u_{2}+\left(\lambda_{3}-\lambda_{2}\right) \gamma_{3} I_{L}=0
\end{gathered}
$$

is replaced $u_{1}=u_{1}^{*}$ y $u_{2}=u_{2}^{*}$ clearing $u_{1}^{*}$ y $u_{2}^{*}$ we obtain

$$
\begin{gathered}
u_{1}^{*}=\frac{1}{A_{1}}\left[\left(\lambda_{2}-\lambda_{1}\right) \beta_{2} S_{L} I_{L}\right] \\
u_{2}^{*}=\frac{1}{A_{2}}\left[\left(\lambda_{2}-\lambda_{3}\right) \gamma_{3} I_{L}\right] q
\end{gathered}
$$




\section{Computational Results}

In this section we have studied numerically the two models - without control and with control for influenza H1N1- from a numerical perspective. An iterative method was used to solve both models with an algorithm in Matlab. The optimal treatment strategy is obtained by solving the optimality system, consisting of ordinary differential equations from the state and adjoint equations. An iterative method is used to solve the optimization problem. We begin to solve the state equations with an estimation for the controls using a forward fourth order Runge-Kutta scheme [15]. By the transversality conditions (9), the adjoint equations are solved by a backward fourth order Runge-Kutta scheme using the current iteration solution of the state equations [15]. Thus, the controls are replaced by using a convex combination of the previous controls and the value given in (10). This process is repeated and is stopped when the values of unknowns at the previous iteration are very close to the values at the present iteration [15].

For the simulations, it is assumed that the weight factor $A_{2}$ associated with control of $u_{2}$ is greater than or equal to $A_{1}$, which is associated with $u_{1}$ control. Here, we assume the following facts: The cost associated with $u_{1}$ includes the cost of screening programs and treatment, and the cost associated with $u_{2}$ includes the cost of keeping patients in the hospital. An infectious person in isolation lasts longer to treat (several weeks) than a person with AH1N1 flu symptoms. The values of the parameters are as follows: $\beta^{*}=0.001, \beta_{1}=$ $0.002, \beta_{2}=0.0013, \gamma_{1}=0.01, \gamma_{2}=0.015, \gamma_{3}=0.01, \mu_{1}=0.001, \mu_{2}=.043$, $\mu_{3}=0.001, \pi=2.5, \Lambda=0.2$.

Figure 5 shows that when applying the treatment effects are on 20, and it takes 8 days to ensure that the condition is stable at low levels. When applied to the isolation of disease it is 10 days and takes 5 days to reach low levels. So if you put more effort into controlling "isolation" case $u_{1}$, the epidemic tends to be controlled quickly and at a lower cost. 


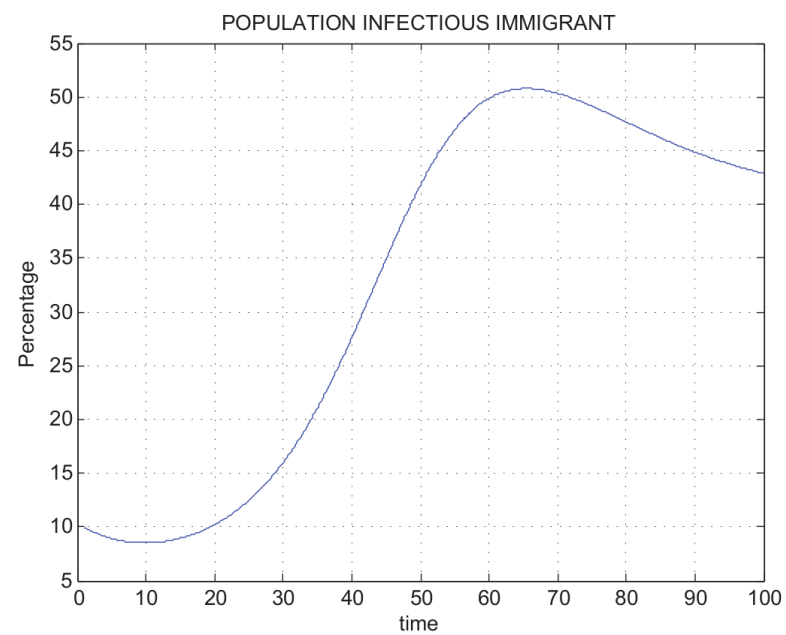

Figure 2: Here we see the variation in the immigrant population infected initially presented in Colombia.

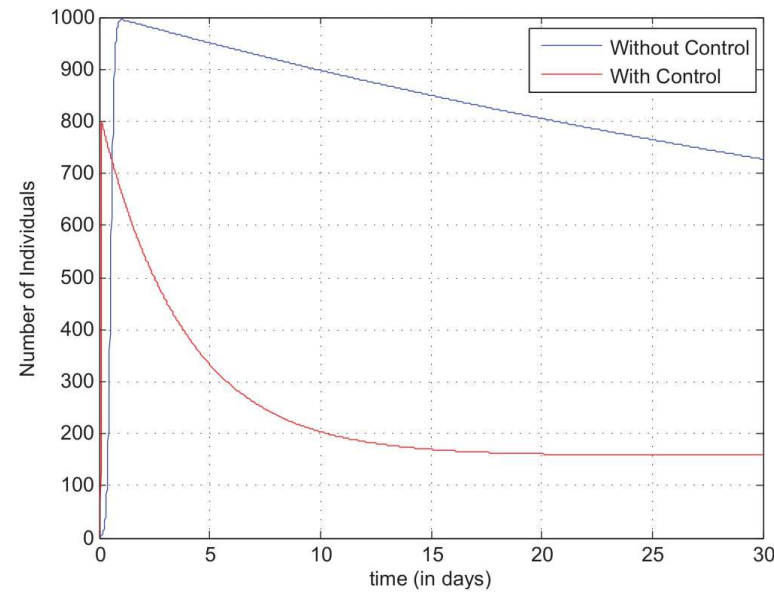

Figure 3: Comparing the populations infected with control and without control. 


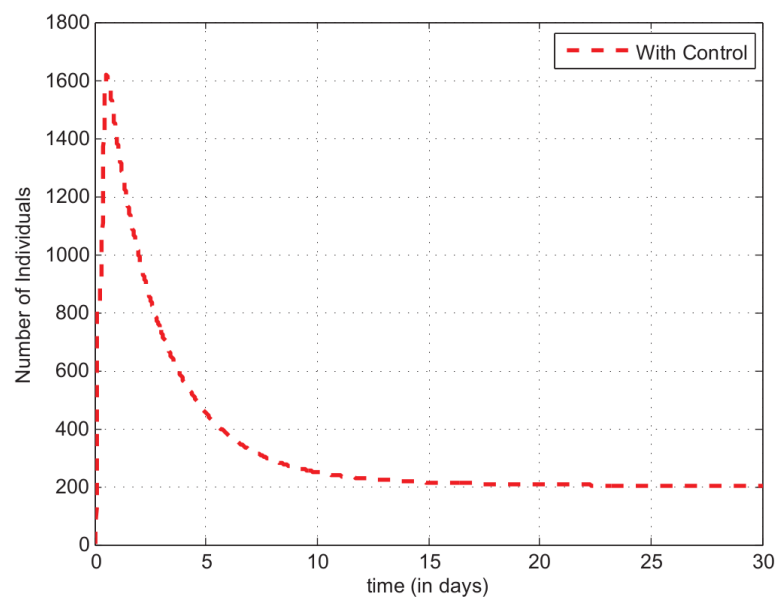

Figure 4: The introduction of control has a positive effect on the infected population.

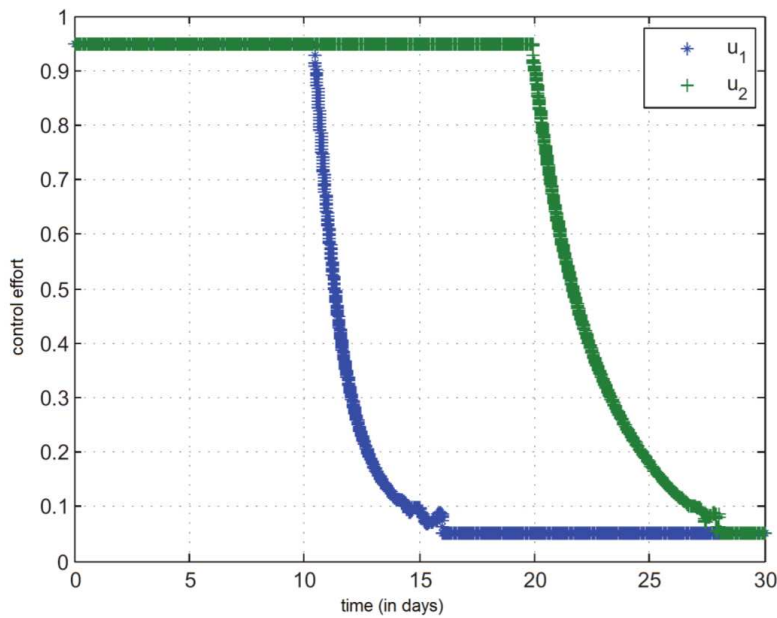

Figure 5: The isolation control is less expensive and more effective than treatment given to patients. 


\section{Discussion and Conclusion}

In this research presents a model to analyze how foreign individuals infected with H1N1 influenza virus caused the outbreak in Colombia. In order to analyze the development of the disease in Colombia, the population has been divided the into two groups: foreign population and local population. This model introduced two time-dependent controls. The first control, $u_{1}(t)$, is applied to the transmission rate $\beta_{2}$ and represents the insulation made of the cases presented. The second control, $u_{2}(t)$, represents the effort put into the treatment of individuals infected with H1N1.

A first important result is summarized in theorem 1, which states that if the basic reproductive number $\mathcal{R}_{E 0}$ for the foreign population in Colombia is less than one and the basic reproductive number for local population $\mathcal{R}_{L 0}$ is also less than one, then $P_{0}$ is the only system equilibrium (1), (2) and is globally asymptotically stable, implying that it is impossible that a large outbreak takes place. Theorem 2 shows that if the basic reproductive number for the local $\mathcal{R}_{L 0}$ is less than one, but the basic reproductive number $\mathcal{R}_{E 0}$ for foreign population is larger than one, then this may be an outbreak.

Control theory helps to determine the maximum benefit or minimum cost of a management plan in an outbreak. Optimal control is used in public health policies to minimize the costs of an epidemic, including patients, deaths, treatments, and others. In our model we have introduced two control strategies to minimize the magnitude of the H1N1 influenza outbreak.

\section{References}

[1] M. Nuno, Mathematical models for the dynamics of influenza at the population and host level. Doctoral Thesis: Cornell University. 2005.

[2] R. B. Couch, J. A. Kasel, Immunity to Influenza in man, Ann. Rev. Micro., 31(1983) $529-549$.

[3] J. R. Davies, E. A. Grilli, A. J. Smith, Influenza A:infection and reinfection, J. Hy., Camb. 92(1984) 125-127.

[4] T.C. Germann, K. Kadau , I.M. Longini ,C.A. Macken . Mitigation strategies for pandemic influenza in the United States. Proc Natl Acad Sci USA 103(2006) 5935-5940.

[5] H. Hethcote. The Mathematics of Infectious Diseases. SIAM Rev. 42(2000) 599-653.

[6] W.H. Hamer. Epidemic Disease in England. Lanc. 1(1906) 733-739.

[7] R. Ross. The prevention of Malaria. (2nd ed., with addendum). John Murray, London (1911).

[8] W.O. Kemarck, A.G. Mackendrick. A contribution to the mathematical theory of epidemics. Proc. R. Soc. Lond. B. 115 (1927) 700-721. 
[9] C. Castillo-Chávez, S. Blower, P. van den Driessche, D. Kirschner, and A-A Yakubu (eds.). Mathematical Approaches for Emerging and Reemerging Infectious Diseases: An Introduction, IMA Vol. 125, Springer-Verlag, USA (2002).

[10] E. De la Pava, B. Salguero, A. Alzate. Modelo Matemático del Riesgo Anual de infección Tuberculosa en Cali. Revista Panamericana de Salud Publica. 11(3)(2002) 166-171.

[11] C. Castillo-Chavez, K. Cooke and S. A. Levin. On the modeling of epidemics. In: High Performance Computing (J. L. Delhaye and E. Gelenbe, eds.). 389-409. North Holland, Amsterdam, New York, Oxford, Tokyo. (1989).

[12] E. De la Pava, B. Salguero. Social mobility and the evolution of tuberculosis. Technical report (2001) Cornell University.

[13] D. Kirschner, S. Lenhart, and S. Serbin. Optimal control of the chemotherapy of HIV, J. Mathematical Biology, 35(1997):775-792.

[14] W. H. Fleming and R. W. Rishel. Deterministic and Stochastic Optimal Control, Springer Verlag, USA (1975).

[15] E. Jun, S. Lenhart, and Z. Feng. Optimal control of treatments in a two-strain tuberculosis model. Discr. Continu. Dynamic. Syst. Series B. 2(4)(2002) 473-482. 
